\title{
Universal screening for hypothyroidism in pregnancy: time for a paradigm shift?
}

\author{
L. D. Premawardhana
}

Received: 3 December 2014/ Accepted: 11 December 2014/Published online: 27 December 2014

(C) Springer Science+Business Media New York 2014

The principles governing screening are well known [1]. But "gut feelings" for the benefits of a screening strategy, are justifiably subjugated to the need for high-quality evidence for its benefits-randomized clinical trials (RCTs) and cost-benefit analyses. As evidence from RCTs is sparse, clinicians continue to debate the benefits of screening strategies even in common disorders such as thyroid disease. Although broad principles are established, often the devil is in the detail. A good example of this is screening for hypothyroidism in pregnancy.

2-3\% of pregnancies are complicated by subclinical hypothyroidism $(\mathrm{SCH})$ and $0.3-0.5 \%$ by overt hypothyroidism $(\mathrm{OvH})$, both autoimmune in iodine-sufficient areas [2]. Women with OvH have 2-3 times higher adverse outcomes compared with euthyroid women and may give birth to neuro-intellectually impaired children. The benefits of treating OvH with thyroxine preconception, and in early pregnancy are established. However, more subtle degrees of thyroid hypofunction such as $\mathrm{SCH}$ and isolated hypothyroxinaemia (IH) are also associated with adverse maternal, fetal, and childhood outcomes. Affected women are asymptomatic and if they and their offspring are to benefit from intervention, they would first need to be identified i.e., some form of screening would be needed. But compared to OvH, the evidence for the effects of and the benefits of treating subtle thyroid hypofunction is less secure. Sixteen observational

\footnotetext{
L. D. Premawardhana $(\bowtie)$

Section of Endocrinology and Diabetes, Department

of Medicine, YYF Hospital, Ystrad Fawr Way,

Caerphilly CF82 7EP, UK

e-mail: premawardhanald@ cardiff.ac.uk

L. D. Premawardhana

Centre for Endocrine and Diabetes Sciences, C2 Link, University

Hospital of Wales, Heath Park, Cardiff CF14 4XN, UK
}

studies to date have failed to demonstrate direct evidence of a consistent relationship probably because they were small studies lacking power to detect abnormal outcomes, with significant variability in the timing of thyroid tests and the definition of thyroid hypofunction. Only one placebo-controlled RCT has shown the benefits of maternal thyroxine treatment in reducing maternal and neonatal adverse outcomes in SCH [3]. Therefore, some authors recommend that screening should primarily attempt to identify OvH (for which there is evidence) rather than subtle hypothyroidism (for which the evidence is incomplete) $[4,5]$.

There is currently no consensus about an optimal screening strategy and the "universal versus targeted screening" debate continues. Specialist Society guidelines about this important subject are at best inconsistent. Only the Spanish Association of Endocrinology and Nutrition (SEEN) and the Indian Thyroid Society (ITS) recommend universal screening. But the Endocrine Society (ES, USA) does not have a consensus view and has proponents on both sides of the screening debate. The American Thyroid Association (ATA) does not recommend universal screening either, but comprehensive patient selection criteria, make their position one of almost tacit approval for universal screening. The American Association of Clinical Endocrinologists (AACE)/ATA guidelines recommend targeted screening. The European Thyroid Association (ETA) guidelines do not recommend universal screening either. However, four of its authors individually support universal screening [6]. Reflecting this uncertainty, practice is variable- only $42 \%$ of members of the ETA-adopted universal screening and $17 \%$ did not screen at all. The figures were 21 and $13 \%$ respectively among Asian clinicians [7].

The meta-analysis by Jouyandeh et al. adds to this debate [8]. They conclude that a significant proportion of pregnant women who have thyroid hypofunction, will remain unidentified if only targeted high-risk screening is 
adopted. The authors only considered studies reporting a "loss ratio" (LR - the percentage of hypothyroid subjects missed when a high-risk case-finding strategy was used) and selected 10 out of 241 studies for further analysis. However, on closer scrutiny, only five of these ten studies reported a LR on screening high-risk pregnant women. Three of ten studies used universal screening and two were, in fact, cost-benefit analyses - these latter five studies were not included in the analysis. The meta-analysis, involving over six thousand subjects, showed LRs between 30 and $81 \%$ with an overall LR of $49 \%$ (CI 0.23-0.74). Unfortunately, there is no indication of the proportion with $\mathrm{OvH}$ among these groups. It is plausible that the definition of "high risk" was variable and accounted at least in part for the wide variability of LR. The cost-benefit analyses concluded that there was a clear advantage to universal screening.

Despite increasing evidence, the principal constraints to a change from targeted to universal screening (Table 1), are based on the lack of demonstrable benefits of detecting subtle thyroid dysfunction-(a) the lack of consistent evidence for the effects of subclinical hypofunction or its treatment on outcomes; and (b) the lack of evidence for beneficial effects on neuro-intellectual outcomes of children of thyroxine-treated mothers. Despite evidence that both SCH and IH affect neuro-intellectual development [911], there is also a scarcity of evidence for the benefits of thyroxine replacement in their prevention. The only RCT hitherto, the CATS study, failed to show a difference in neuro-intellectual outcomes in children at 3 years of age, born to untreated mothers, and to mothers given thyroxine for SCH and IH [12]. This lack of effect may have been due to the timing of maternal thyroxine initiation, and also of neuro-intellectual testing of children.

Given these confusing data and recommendations, what should a practising clinician do? Many experts and some

Table 1 National and regional specialist society recommendations for thyroid screening in pregnancy

\begin{tabular}{llc}
\hline $\begin{array}{l}\text { Specialist } \\
\text { society }\end{array}$ & Recommended screening strategy & Year \\
\hline ACOG & High-risk targeted & 2007 \\
ATA & High-risk targeted & 2011 \\
ES (USA) & High-risk targeted & 2012 \\
AACE & Universal screening not recommended. High- & 2012 \\
& $\quad$ risk targeted screening not addressed & \\
SEEN & Universal screening & 2012 \\
ITS & Universal screening & 2012 \\
AACE/ATA & High-risk targeted & 2012 \\
ETA & High-risk targeted & 2014 \\
\hline
\end{tabular}

Abbreviations for specialist societies-see text for explanation professional societies believe that it is time to consider a paradigm shift in screening strategy. They contend that universal screening is the most cost-effective option. The uncertainty about $\mathrm{SCH}$ and $\mathrm{IH}$ notwithstanding, the detection of $\mathrm{OvH}$ alone (where there is good evidence for the benefits of intervention) makes the exercise worthwhile. Recently, Pop et al. showed that in 2012 among healthy Caucasian Dutch women, an estimated $0.62 \%$ had OvHextrapolated country wide, 992 women would have been detected with $\mathrm{OvH}$ or elevated TSH levels - the estimated figures being 4,166 for the UK and 24,800 for USA [13]. As mentioned above, there is strong evidence for adverse pregnancy and childhood neuro-intellectual developmental outcomes in untreated OvH. There is also good evidence that thyroxine treatment of affected women prevents these. It is time to support a paradigm shift to universal screening because-(a) it would identify the significant numbers of women with $\mathrm{OvH}$ who would remain unidentified with targeted high-risk screening; (b) maternal and fetal morbidity and childhood neuro-intellectual abnormalities associated with untreated $\mathrm{OvH}$ may be ameliorated; (c) and these would be done in a cost-effective way.

The current parlous state of RCT evidence for the benefits of universal screening, should not be interpreted as evidence against it. More well-designed studies are required, about the timing of screening and intervention, thresholds for treatment, and the logistics of a universal screening program e.g., who will screen, interpret, and respond to test results, follow up, and dose titrate, etc. But it would be imprudent to be inactive in such a "screen friendly" condition, till the evidence is beyond reproach.

\section{References}

1. N. Wald, M. Law, Medical Screening, in Oxford Textbook of Medicine, 5th edn., ed. by D.A. Warrell, T.M. Cox, J.D. Firth (Oxford University Press, Oxford, 2010), pp. 94-108

2. R. Moreno-Reyes, D. Glinoer, H. Van Oyen, S. Vandevijvere, High prevalence of thyroid disorders in pregnant women in a mildly iodine-deficient country: a population-based study. J. Clin. Endocrinol. Metab. 98, 3694-3701 (2013)

3. R. Negro, A. Schwartz, R. Gismondi, A. Tinelli, T. Mangieri, A. Stagnaro-Green, Universal screening versus case finding for detection and treatment of thyroid hormonal dysfunction during pregnancy. J. Clin. Endocrinol. Metab. 95, 1699-1707 (2010)

4. P. Laurberg, L. Stine, S. Andersen, I.B. Pedersen, S. Andersen, A. Carle, Screening for overt thyroid disease in early pregnancy may be preferable to searching for small aberrations in thyroid function tests. Clin. Endocrinol. 79, 297-304 (2013)

5. R. Negro, A. Stagnaro-Green, Diagnosis and management of subclinical hypothyroidism in pregnancy. Br. Med. J. 349, g4929 (2014)

6. J. Lazarus, R.S. Brown, C. Daumerie, A. Hubalewska-Dydejczyk, R. Negro, B. Vaidya, 2014 European thyroid association guidelines for the management of subclinical hypothyroidism in pregnancy and in children. Eur. Thyroid J. 3, 76-94 (2014) 
7. F. Azizi, A. Amouzegar, L. Mehran, S. Alamdari, I. Subekti, B. Vaidya, K. Poppe, T.S. Luis Jr, T. Akamizu, Screening and management of hypothyroidism in pregnancy: results of an Asian survey. Endocr. J. 61, 697-704 (2014)

8. Z. Jouyandeh, S. Hasani-Ranjbar, M. Qorbani, B. Larijani, Universal screening versus selective case-based screening for thyroid disorders in pregnancy. Endocrine (2014). doi:10.1007/s12020014-0385-9

9. J.E. Haddow, G.E. Palomaki, W.C. Allan, J.R. Williams, G.J. Knight, J. Gagnon et al., Maternal thyroid deficiency during pregnancy and subsequent neuropsychological development of the child. N. Engl. J. Med. 341, 549-555 (1999)

10. V.J. Pop, E.P. Brouwers, H.L. Vader, T. Vulsma, A.L. van Baar, J.J. de Vijlder, Maternal hypothyroxinaemia during early pregnancy and subsequent child development: a 3-year follow-up study. Clin. Endocrinol. 59, 282-288 (2003)

11. J. Henrichs, J.J. Bongers-Schokking, J.J. Schenk, A. Ghassabian, H.G. Schmidt, T.J. Visser et al., Maternal thyroid function during early pregnancy and cognitive functioning in early childhood: the generation R study. J. Clin. Endocrinol. Metab. 95, 4227-4234 (2010)

12. J.H. Lazarus, J.P. Bestwick, S. Channon, R. Paradice, A. Maina, R. Rees et al., Antenatal thyroid screening and childhood cognitive function. N. Engl. J. Med. 366, 493-501 (2012)

13. V. Pop, M. Broeren, W. Wiersinga, The attitude towards hypothyroidism during early gestation: time for a change of mind? Thyroid 24, 1541-1546 (2014) 